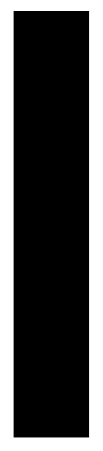

\title{
Palatal Dimensions and Its Correlation with the Circumference of Upper Ante- rior Teeth
}

Zeina M Ahmad
BDS, MSc (Lect)
Department of Prosthetic Dentistry
College of Dentistry, University of Mosul

\begin{abstract}
Aims: To put a bases for the prediction of the circumference of artificial anterior teeth depending on the palatal dimensions Materials and methods: The study sample consists of 30 female and 32 male with class I occlusion aged 19- 24 years.The dimensions of the palatal vault and the circumferences of upper anterior teeth were recorded with digital venire caliper and analyzed with SPSS program (version 11). Results: significant correlation was found between the dimension of the palatal vault and the circumference of upper anterior teeth. Conclusions: the angle of circumferences of upper anterior teeth of narrow short and deep palate will be more tapered than those one in the wide, long and shallow palate.

Keyword: Circumferences of upper anterior teeth ,palatal dimensions.
\end{abstract}

Ahmad ZM. Palatal Dimensions and Its Correlation with the Circumference of Upper Anterior Teeth. Al-Rafidain Dent J. 2009; 9(2): 259- 267.

Received: 2/6/2008 Sent to Referees: $3 / 6 / 2008 \quad$ Accepted for Publication:28/8/2008

\section{INTRODUCTION}

The ultimate support of the maxillary denture is the bone of two maxilla and palatine bone. They form the foundation for the hard palate and provide considerable support for the denture .Hard palate is examined for its shape, height, width and the quality of mucosa and submucosa covering it, high and wide palatal vault will resist lateral displacement of the denture and supply denture support and surface area for retention ${ }^{(1)}$.

Palatal dimensions are statically different between sex and race, ${ }^{(2-5)}$ so that, there is opportunity to establish criteria by which the dentist and forensic physician can predict the race and sex of unknown individual furthermore the palatal dimension may form a basis for identification of many disease like Marfan and Downs syndrome ${ }^{(6,7)}$.

The form of palatal vault give an idea to the original form to the dental arch before the removal of the natural teeth and resorption of residual ridge ${ }^{(1)}$. Ideal relationship between the denture and facial soft tissue provide the basis for the analyzing the beauty and smile ${ }^{(8-10)}$. The circumference of upper anterior teeth is significantly affect the position and expression of the lip and the lower part of the face ${ }^{(11)}$,insufficient lip support resulting from the lower teeth locating too far posteriorly is characterized by dropping of the corner of the mouth ,reduction in the visible part of the vermilon border of the lip ,deepings of nasolable groove, wrinkles above the vermilon border ,deepings of sulci and reduction in prominence of philitrum ${ }^{(1)}$ .the purpose of this study was to study the palatal dimensions and to determine the circumference of upper anterior teeth that depends on the palatal dimensions.

\section{MATERIALS AND METHODS}

The dimension and shape of hard palatal tissue in 30 male and 32 female age range between (19-24) year,all with complete permanent dentition CI.I molar relation were analyzed ,subject with respiratory problem ,previous history of craniofacial truma, congenital anomalies, surgery or orthodontic treatment were not included in the sample .

The upper dental arch and the hard palatal tissue were reproduced from alginate impression casted in detal stone.The mesial 
contact point of two central incisor and the palatal and mesiopalatal cusp tip of posterior teeth were identified with line lead pencil to facilitate identification .All the measurements were carried out using electronic digital vernier caliper (LEZACO, ART, 27710150 MM accuracy, China) .The measurements were recorded with two decimals.The dimension of hard palatal tissue were identified by analyzing the following measurements:
The width of palate is measured between the palatal cusp tip of the two selected teeth. the buccal cusp is not included in the study to exclude the possible variation of the width of the of the teeth that found in and between the two sex which may be source of bias in the measurements ${ }^{(12-15)}$. It include the following distances (Figure 1):

1-Inter first premolar width (4-4)

2- Inter second premolar width (5-5)

3- Inter first molar width (6-6)

4- Inter second molar width (7-7)

\section{A-palatal width}

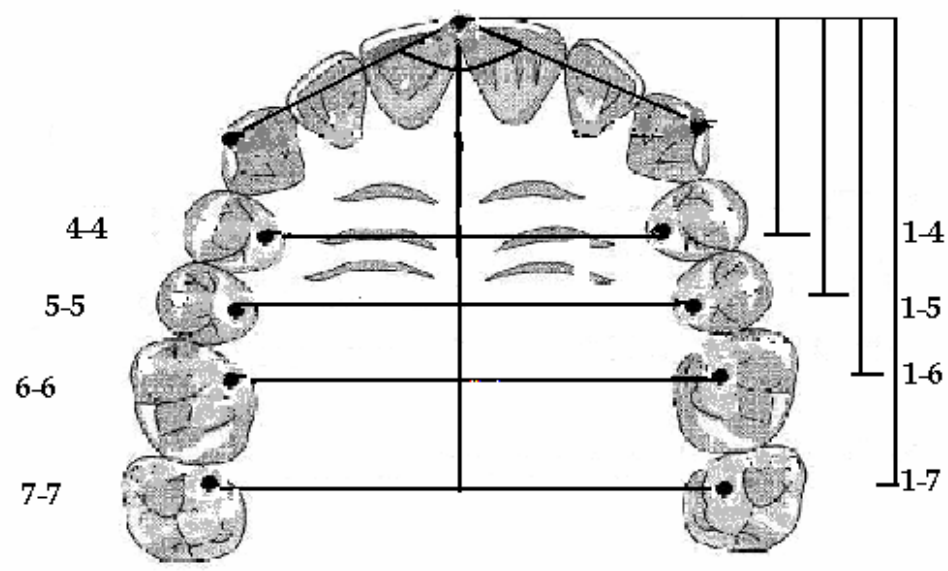

Notes: 4-4: inter first premolar width, 5-5: inter second premolar width, 6-6: inter first molar width, 7-7: inter second molar width,1-4: inter first premolar length,1-5: intersecond premolar length ,1-6: inter first molar length ,1-7: inter second molar length, circum: angle of circumference of upper anterior teeth.

Figure (1): Width and length of palatal vault and anterior arch circumference.

\section{B-Length of the palate}

It is represented by the linear distance from mesial contact point of upper central incisor to the mid point of linear distance between the two selected teeth ,it include the following distance (Figure 1):

1 -Inter first premolar length (1-4)

2 - Inter second premolar length (1-5)

3 - Inter first molar length (1-6)

4- Inter second molar length (1-7)

\section{C-palatal depth :}

The palatal depth is represented by vertical distance from the mid point of the linear distance between the palatal cusp of the two selected teeth to the deepest point of the mid palatal suture measured by the vertical traveling arm of the venire, it include the following linear measurements (Figure 2) :

1-Inter first premolar depth (4-p)

2 - Inter second premolar depth (5-p)

3 - Inter first molar depth (6-p)

4- Inter second molar depth (7-p) 


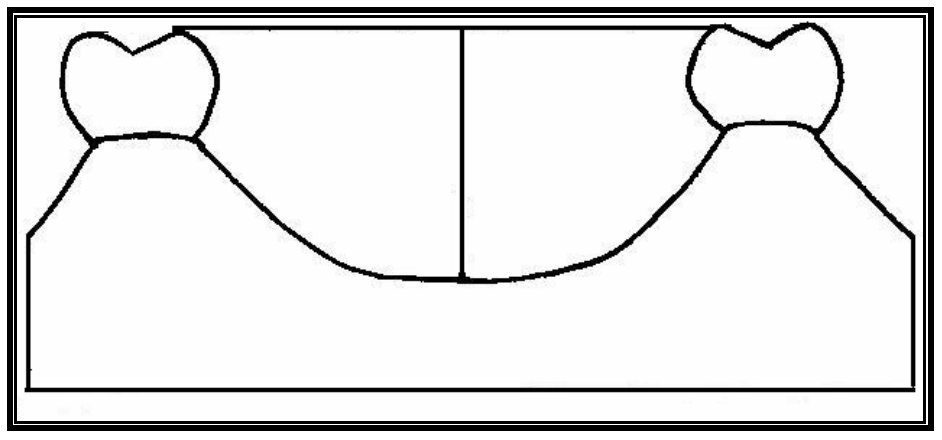

Figure (2): Depth of the palatal vault

D-Circumferences of upper anterior teeth

The width of upper anterior teeth and the canine arc distance is significantly different in and between the two sexes ${ }^{(16-18)}$,so the circumference of upper anterior teeth was measured as the angle formed between the linear distance from the point mid way between the two central incisors to the cusp tip of right and left canine, the angle(which represents the circum) was drawn on graphic paper and measured using protractor, (Figure 1).

The measurements of the palatal arch dimensions were analyzed with statistical package for social science (spss, version 11) for production of means, standard division, $\mathrm{t}$ - test and the coefficient of correlation between the recorded variable.

\section{RESULTS}

The descriptive statistics of the palatal arch dimensions (width, length and depth) and the circumference of upper anterior were obtained, Tables $(1,2)$. The width of palatal vault anteriorly is $32.32 \mathrm{~mm}, 15.38 \mathrm{~mm}$ ,13.29 mm (width ,length and depth) and increasing to $41.47,36.23$ and $20.1 \mathrm{~mm}$ posteriorly adjacent to first molars. The average circumference of upper anterior teeth was 126.1 degree.

Table (1) : Descriptive statistics of palatal dimensions

\begin{tabular}{ccccc}
\hline Varibles & Minimum & Maximum & Mean & Std. Deviation \\
\hline $4-4$ & 25.68 & 39.44 & 32.3203 & 2.57721 \\
$5-5$ & 28.75 & 46.00 & 36.9275 & 2.95600 \\
$6-6$ & 36.00 & 47.25 & 41.4767 & 2.35906 \\
$7-7$ & 36.32 & 54.01 & 47.1364 & 3.00150 \\
$1-4$ & 12.72 & 18.96 & 15.3881 & 1.71857 \\
$1-5$ & 17.11 & 25.92 & 21.6362 & 2.03743 \\
$1-6$ & 31.00 & 44.22 & 36.2320 & 2.34436 \\
$1-7$ & 36.18 & 52.19 & 44.8219 & 2.85609 \\
$4-p$ & 6.25 & 19.05 & 13.2911 & 2.96025 \\
$5-p$ & 11.25 & 24.79 & 18.0919 & 2.98049 \\
$6-p$ & 14.91 & 26.14 & 20.1025 & 2.59635 \\
$7-p$ & 15.05 & 25.50 & 19.4083 & 2.44822 \\
\hline
\end{tabular}

4-4:Inter first premolar width, 5-5: Inter second premolar width, 6-6: Inter first molar width, 7-7: Inter second molar width, 1-4: Inter first premolar length , 1-5: Inter second premolar length, 1-6: Inter first molar length, 1-7: Inter second molar length, 4-p: Inter first premolar depth, 5-p: Inter second premolar depth , 6-p: Inter first molar depth , 7-p: Inter second molar depth. 
Table (2) : Descriptive statistics of circumference of anterior teeth

\begin{tabular}{ccccc}
\hline Variables & Minimum & Maximum & Mean & Standard Deviation \\
\hline Circum & 110.00 & 145.00 & 126.1094 & 7.34656 \\
\hline \multicolumn{2}{c}{ Circum: Angle of circumference of upper anterior teeth. }
\end{tabular}

Tables $(3,4)$ reveled that the collected measurements of the palatal dimesions and the angle of circumference of upper anterior teeth in the males group is larger than those measurements in the females group this difference is not significant except the palatal depth of males is significantly higher than females.

Table (3) : Paired -sample T test for the palatal dimensions (male, female)

\begin{tabular}{|c|c|c|c|c|}
\hline Variables & Sex & Mean & Standard Deviation & $\mathbf{T}$-value \\
\hline \multirow{2}{*}{$4-4$} & male & 32.5293 & 203872 & \multirow{2}{*}{1.378} \\
\hline & female & 31.6661 & 2.7665 & \\
\hline \multirow{2}{*}{$5-5$} & male & 37.9118 & 3.6660 & \multirow{2}{*}{3.162} \\
\hline & female & 35.6768 & 2.6820 & \\
\hline \multirow{2}{*}{$6-6$} & male & 42.1818 & 2.0746 & \multirow{2}{*}{3.246} \\
\hline & female & 40.3914 & 2.0647 & \\
\hline \multirow{2}{*}{$7-7$} & male & 47.8521 & 3.263 & \multirow{2}{*}{2.684} \\
\hline & female & 45.9825 & 2.0763 & \\
\hline \multirow{2}{*}{$1-4$} & male & 15.5223 & 1.76099 & \multirow{2}{*}{0.025} \\
\hline & female & 15.0220 & 2.45132 & \\
\hline \multirow{2}{*}{$1-5$} & male & 22.1937 & 2.10109 & \multirow{2}{*}{0.025} \\
\hline & female & 21.3796 & 1.86717 & \\
\hline \multirow{2}{*}{$1-6$} & male & 36.5639 & 1.39963 & \multirow{2}{*}{2.3444} \\
\hline & female & 36.0254 & 1.55707 & \\
\hline \multirow{2}{*}{$1-7$} & male & 45.1100 & 2.10109 & \multirow{2}{*}{2.8561} \\
\hline & female & 44.9000 & 1.86717 & \\
\hline \multirow{2}{*}{$4-p$} & male & 14.1997 & 3.08715 & \multirow{2}{*}{$2.008^{*}$} \\
\hline & female & 12.5623 & 2.71583 & \\
\hline \multirow{2}{*}{$5-\mathrm{p}$} & male & 19.4653 & 2.84811 & \multirow{2}{*}{$3.444^{*}$} \\
\hline & female & 16.8330 & 2.71896 & \\
\hline \multirow{2}{*}{$6-p$} & male & 21.3867 & 2.65394 & \multirow{2}{*}{$3.884 *$} \\
\hline & female & 18.7823 & 1.97354 & \\
\hline \multirow{2}{*}{ 7-p } & male & 20.5880 & 2.45132 & \multirow{2}{*}{$4.287 *$} \\
\hline & female & 18.0830 & 1.85126 & \\
\hline
\end{tabular}

4-4: Inter first premolar width, 5-5: Inter second premolar width, 6-6: Inter first molar width, 7-7: Inter second molar width, 1-4: Inter first premolar length , 1-5: Inter second premolar length, 1-6: Inter first molar length, 1-7: Inter second molar length, 4-p: Inter first premolar depth, 5-p: Inter second premolar depth, 6-p: Inter first molar depth, 7-p: Inter second molar depth, , *:significant difference.

Table (4) : Paired -sample T test for the anterior arch circumference(male ,female)

\begin{tabular}{ccccc}
\hline variables & sex & Mean & Std. Deviation & T-value \\
\hline \multirow{2}{*}{ circum } & male & 126.3000 & 8.08639 & 0.240 \\
& female & 125.8333 & 7.02745 & \\
\hline
\end{tabular}

Circum: circumference of upper anterior teeth 
The palatal arch dimensions is positively correlated to palatal length except in the area adjacent to first molars is significantly reversed Table (5) while the width of palate is mainly reversely correlated to the depth especially in the area adjacent to first molars and this depth is diagonally correlated to palatal length.
The correlation between the palatal arch dimensions and the circumferences of upper anterior teeth in both sexes shown in Tables (6-8). The circumferences of upper anterior teeth is transversally correlated to the width of the palatal vault at the area adjacent to first molar, while it inversely correlated to the length and depth of palatal vault.

Table (5) : Correlations of palatal arch dimensions to each other

\begin{tabular}{|c|c|c|c|c|c|}
\hline Variables & Sex & 4-4 & $5-5$ & 6-6 & $7-7$ \\
\hline \multirow{3}{*}{$1-4$} & female & 0.227 & 0.075 & -0.014 & 0.249 \\
\hline & male & $0.395(*)$ & $0.714(* *)$ & $-0.472(* *)$ & $0.660(* *)$ \\
\hline & total & 0.200 & $0.302(*)$ & 0.288(*) & 0.004 \\
\hline \multirow{3}{*}{$1-5$} & female & $0.670(* *)$ & $0.794(* *)$ & $-0.574(* *)$ & $0.548(* *)$ \\
\hline & male & $0.736(* *)$ & $0.492(* *)$ & $-0.375(*)$ & $0.519(* *)$ \\
\hline & total & $0.317(*)$ & 0.151 & $0.342(*)$ & 0.050 \\
\hline \multirow{3}{*}{$1-6$} & female & $0.714(* *)$ & $0.557(* *)$ & -0.187 & $0.875(* *)$ \\
\hline & male & $0.578(* *)$ & $0.704(* *)$ & -0.283 & $0.851(* *)$ \\
\hline & total & $0.383(* *)$ & $0.366(* *)$ & $0.427(* *)$ & $0.267(*)$ \\
\hline \multirow{3}{*}{$1-7$} & female & $0.670(* *)$ & $0.794(* *)$ & $-0.574(* *)$ & $0.548(* *)$ \\
\hline & male & $0.736\left(^{* *}\right)$ & $0.492(* *)$ & $-0.375(*)$ & $0.519(* *)$ \\
\hline & total & $0.431(* *)$ & $0.347(* *)$ & $0.436(* *)$ & $0.258(*)$ \\
\hline \multirow[t]{3}{*}{ 4-p } & female & $0.414(*)$ & $0.481(* *)$ & $-0.457(*)$ & $0.409(*)$ \\
\hline & male & 0.279 & $0.464(* *)$ & $-0.590(* *)$ & 0.164 \\
\hline & total & -0.109 & 0.061 & 0.200 & 0.221 \\
\hline \multirow{3}{*}{ 5-p } & female & 0.216 & 0.346 & $-0.416(*)$ & 0.241 \\
\hline & male & 0.143 & $0.373(*)$ & $-0.530(* *)$ & 0.013 \\
\hline & total & -0.116 & 0.069 & 0.085 & 0.163 \\
\hline \multirow[t]{3}{*}{ 6-p } & female & 0.293 & $0.362(*)$ & $-0.474(* *)$ & 0.196 \\
\hline & male & 0.241 & $0.486(* *)$ & $-0.594(* *)$ & 0.156 \\
\hline & total & -0.054 & 0.098 & 0.167 & 0.211 \\
\hline \multirow[t]{3}{*}{ 7-p } & female & 0.214 & 0.139 & -0.247 & -0.022 \\
\hline & male & 0.170 & $0.512(* *)$ & $-0.605(* *)$ & 0.159 \\
\hline & total & -0.105 & 0.085 & 0.110 & 0.147 \\
\hline Variables & sex & $1-4$ & 1-5 & 1-6 & 1-7 \\
\hline \multirow[t]{3}{*}{ 4-p } & female & -0.110 & -0.222 & -0.184 & -0.089 \\
\hline & male & $0.464(* *)$ & $0.373(*)$ & $0.486(* *)$ & $0.512(* *)$ \\
\hline & total & $.349(* *)$ & 0.233 & 0.265 & 0.248 \\
\hline \multirow[t]{3}{*}{ 5-p } & female & 0.361 & 0.036 & 0.077 & 0.090 \\
\hline & male & $0.480(* *)$ & 0.267 & $0.419(*)$ & 0.355 \\
\hline & total & $0.447(* *)$ & 0.206 & $0.316(*)$ & $0.283(*)$ \\
\hline \multirow{3}{*}{ 6-p } & female & 0.296 & 0.133 & 0.137 & 0.017 \\
\hline & male & 0.150 & 0.161 & 0.218 & 0.232 \\
\hline & total & $0.357(* *)$ & 0.152 & $0.283(*)$ & 0.229 \\
\hline \multirow{3}{*}{ 7-p } & female & 0.361 & 0.036 & 0.077 & 0.090 \\
\hline & male & $0.480(* *)$ & 0.267 & $0.419(*)$ & 0.355 \\
\hline & total & $0.318(*)$ & 0.153 & 0.222 & 0.154 \\
\hline
\end{tabular}

4-4: Inter 1st premolar width, 5-5: Inter 2nd premolar width, 6-6: Inter 1st molar width, 7-7: Inter 2nd molar width, 1-4: Inter 1st premolar length, 1-5: Inter 2nd premolar length, 1-6: Inter 1st molar length , 1-7: Inter 2nd molar length, 4-p:inter 1st premolar depth, 5-p: Inter 2nd premolar depth, 6-p: Inter 1st molar depth , 7-p: Inter 2nd molar depth , ${ }^{* *}$ Correlation s is significant at the 0.01 
Table (6): Correlations of palatal width to anterior arch circumference

\begin{tabular}{|c|c|c|c|c|c|}
\hline Variables & Sex & 4-4 & $5-5$ & 6-6 & $7-7$ \\
\hline \multirow{3}{*}{$4-4$} & female & & & & \\
\hline & male & & & & \\
\hline & total & & & & \\
\hline \multirow{3}{*}{$5-5$} & female & $0.594(* *)$ & & & \\
\hline & male & $0.461(* *)$ & & & \\
\hline & total & $0.637(* *)$ & & & \\
\hline \multirow{3}{*}{ 6-6 } & female & -0.352 & $-0.830(* *)$ & & \\
\hline & male & -0.256 & $-0.785(* *)$ & & \\
\hline & total & $0.678(* *)$ & $0.702(* *)$ & & \\
\hline \multirow{3}{*}{$7-7$} & female & $0.633(* *)$ & $0.584(* *)$ & -0.203 & \\
\hline & male & $0.628(* *)$ & $0.698(* *)$ & -0.303 & \\
\hline & total & $0.339(* *)$ & $0.435(* *)$ & $0.670(* *)$ & \\
\hline \multirow{3}{*}{ circum } & female & -0.352 & $-0.830(* *)$ & $1.000(* *)$ & -0.203 \\
\hline & male & -0.256 & $-0.785(* *)$ & $1.000(* *)$ & -0.303 \\
\hline & total & 0.221 & 0.154 & 0.029 & -0.108 \\
\hline
\end{tabular}

4-4: Inter first premolar width, 5-5: Inter second premolar width, 6-6: Inter first molar width, 7-7: Inter second molar width, Circum: Angle of circumference of upper anterior teeth, **: Correlation is significant at the 0.01 level , *: Correlation is significant at the 0.05 level.

Table 7 : Correlations of palatal length to anterior arch circumference

\begin{tabular}{|c|c|c|c|c|c|}
\hline Variables & Sex & 1-4 & $1-5$ & 1-6 & $1-7$ \\
\hline $1-4$ & $\begin{array}{c}\text { female } \\
\text { male } \\
\text { total }\end{array}$ & & & & \\
\hline \multirow{3}{*}{$1-5$} & female & 0.200 & & & \\
\hline & male & $0.403(*)$ & & & \\
\hline & total & $0.561(* *)$ & & & \\
\hline \multirow{3}{*}{ 1-6 } & female & 0.187 & $0.612(* *)$ & & \\
\hline & male & $0.573(* *)$ & $0.434(*)$ & & \\
\hline & total & $0.703(* *)$ & $0.484(* *)$ & & \\
\hline \multirow{3}{*}{ 1-7 } & female & 0.200 & $1.000(* *)$ & $0.612(* *)$ & \\
\hline & male & $0.403(*)$ & $1.000(* *)$ & $0.434(*)$ & \\
\hline & total & $0.761(* *)$ & $0.504(* *)$ & $0.836(* *)$ & \\
\hline \multirow{3}{*}{ circum } & female & -0.014 & $-0.574(* *)$ & -0.187 & $-0.574(* *)$ \\
\hline & male & $-0.472(* *)$ & $-0.375\left(^{*}\right)$ & -0.283 & $-0.375(*)$ \\
\hline & total & $-0.432(* *)$ & $-0.500(* *)$ & $-0.291\left(^{*}\right)$ & $-0.336(* *)$ \\
\hline
\end{tabular}

1-4: Inter first premolar length , 1-5: Inter second premolar length, 1-6: Inter first molar length , 1-7: Inter second molar length, Circum: Angle of circumference of upper anterior teeth, ** : Correlation is significant at the 0.01 level, *: Correlation is significant at the 0.05 level 
Table (8) : Correlations of palatal depth and anterior arch circumference

\begin{tabular}{|c|c|c|c|c|c|}
\hline Variables & Sex & 4-p & 5-p & 6-p & 7-p \\
\hline 4-p & $\begin{array}{l}\text { female } \\
\text { male } \\
\text { total }\end{array}$ & & & & \\
\hline 5-p & $\begin{array}{l}\text { female } \\
\text { male } \\
\text { total }\end{array}$ & $\begin{array}{l}0.834(* *) \\
0.765(* *) \\
0.801(* *)\end{array}$ & & & \\
\hline 6-p & $\begin{array}{l}\text { female } \\
\text { male } \\
\text { total }\end{array}$ & $\begin{array}{l}0.689(* *) \\
0.666(* *) \\
0.686(* *)\end{array}$ & $\begin{array}{l}0.856(* *) \\
0.832(* *) \\
0.864(* *)\end{array}$ & & \\
\hline $7-\mathbf{p}$ & $\begin{array}{l}\text { female } \\
\text { male } \\
\text { total }\end{array}$ & $\begin{array}{l}0.474\left(^{* *}\right) \\
0.526(* *) \\
0.541\left(^{* *}\right)\end{array}$ & $\begin{array}{l}0.663(* *) \\
0.758(* *) \\
0.768(* *)\end{array}$ & $\begin{array}{l}0.823(* *) \\
0.866(* *) \\
0.887(* *)\end{array}$ & \\
\hline circum & $\begin{array}{l}\text { female } \\
\text { male } \\
\text { total }\end{array}$ & $\begin{array}{c}-0.457(*) \\
-0.590(* *) \\
-0.511(* *)\end{array}$ & $\begin{array}{c}-0.416\left(^{*}\right) \\
-0.530\left(^{* *}\right) \\
-0.425\left(^{* *}\right)\end{array}$ & $\begin{array}{l}-0.474\left(^{* *}\right) \\
-0.594(* *) \\
\left.-0.4611^{* *}\right)\end{array}$ & $\begin{array}{c}-0.247 \\
-0.605(* *) \\
-0.381\left(^{* *}\right)\end{array}$ \\
\hline
\end{tabular}

4-p: Inter first premolar length, 5-p: Inter second premolar length, 6-p: Inter first molar length, 7-p: Inter second molar length, Circum: Angle of circumference of upper anterior teeth. **: Correlation is significant at the 0.01 level, *: Correlation is significant at the 0.05 level.

\section{DISCUSSION}

In this study it was obvious that the measurements taken for the dimensions of anterior palatal vault is smaller than the posterior palatal vault this confirm the natural view that the posterior palatal segment is wider and deeper than the anterior one.

$\mathrm{T}$ - test revealed that there is appreciable sex difference in the dimensions of the palatal vault ,the size of palate is larger in males group than females and the average difference in the palatal depth is significantly higher in males than females this simply reflect the greater overall breadth of male skull in any given ethnic group ${ }^{(19)}$,these results is in agreement with the result of other previous studies ${ }^{(20-24)}$. Table (2) revealed that the angle of the circumferences of upper anterior teeth in females (123 degree) is smaller than those in males group (126 degree), this result come in accordance with the natural view that the feminity is characterized by curved surfaces roundness and softness in the form of dentition while the masculinity is characterized by boldness ,vigor and squrness in dentition ${ }^{(1)}$.

Different correlations were found among the palatal arch dimensions (Table 5) ,generally the correlation between the palatal width and length is significantly positive except at the inter first molar distance the is significantly negative i.e as the palatal arch length increase the width is also increase until the inter first molar the width is no longer increase. The directly propotional correlation between the width and length of palatal vault anteriorly until the first molar were the correlation was reversed, this result give rise to the accepted natural view that the shape of palatal arch is $U$ shape palatal vault $^{(1)}$ other wise the shape of palate will be semicircular.

The width of palate as measured from the palatal and mesiopalatal cusp tip of teeth is mainly negatively correlated to palatal depth specially at the inter first molar area i.e the wide palate is characterized by shallow palatal vault. The result indicated also that the 
increase length of palate is accompanied by increasing palatal depth. On other hand the angle of circumference o $\mathrm{f}$ upper anterior teeth is positively correlated to the width of posterior palatal vault and reversely correlated to the length and depth of palate these result confirm the accepted view that the broad and shallow edentulous palatal vault indicated that the dental arch form originally been square and a high $\mathrm{V}$ shape edentulous vault indicated a tapering dental arch form , rounded vault of average height may indicate an ovoid dental arch ${ }^{(!)}$, these finding is of practical importance to prosthodontist as it form the basis for the arrangements of artificial upper anterior teeth so that they can previously predict the circumferences of anterior teeth by observing the dimensions of palatal vault i.e the arc of circumferences of the artificial anterior teeth of the narrow edentulous arch as measured from the crest of residual ridge will be more tapered than those of the wide edentulous arch.

\section{CONCLUSIONS}

The anterior palatal vault is smaller than the posterior one

The dimensions of the palatal vault in males is larger than females especially the palatal depth

The angle of circumference of upper anterior teeth as measured from the measial contact point of the two central incisors and the cusp tip of canines in females is more tapered than males

Wide palatal vault as measured from the palatal and mesiopalatal cusp tip of teeth is mainly characterized by long and shallow palate.

The angle of circumferences of upper anterior teeth of the wide ,long and shallow palate will be less tapered than those one in the narrow short and deep palate .

\section{REFERENCES}

1.Zarb GA, Blonder CL ,Carlsson GE .Boucher's Prosthodontic treatment for edentulous patient ,eleventh edition, copy right by Mosby-year book, Inc St.louis,Missouri 1997,57-87.

2.Byers SN,Churchill SE ,Gurran B.Identification of Euro-Americans,Afro-Americans and Amerindians from the palatal dimensions. J For Sci .1997;42:3-9.

3.Hohoff A ,Rabe H, Ehmer U,Harm E.Gender . Palatal development of preterm and low birth weight infants compared to term infants .what do we know?part1: the palate of the term newborn. Head and face medicine .2005;1:8.

4.Burris BG.Identification of race and sex from the palatal dimension. $J$ Forensic Sci .1998; 43(5):959-63.

5.Friend GW, Harris EF ,Mincer HH, Fong TL ,Garruth K. Oral anomalies in the neonate by race and gender in an urban setting. Pediat Dent . 1990; 12:157-67.

6. Westling L, Mohlin B, Bresin A. Crainofacial manifestation in Marfan syndrome: palatal dimensions and comparative cephalometric analysis's. Craniofac Dev Biol. 1998;18(4):211-80.

7.Westrerman G, Johnson R , Cohen MM. Variation of palatal dimensions in patient with downs syndrom. $J$ Dent Res .1975;54(4):767-71.

8. Holdaway RA. A soft tissue cephalometric analysis and its use in orthodontic treatment planning part 1. Am J orthod.1983;84:1-28.

9.-Arnett GW, Bergman RT .Facial keys to orthodontic diagnosis and treatment planning. part 1.Am J orthod Dentofac orthop .1993:103:299-312.

10. Arnett GW, Bergman RT .Facial keys to orthodontic diagnosis and treatment planning. part 11.Am J orthod Dentofac orthop .1993 :103:395-411.

11. Mack MR. Perspective facial esthetic in dental treatment planning. JProsthet Dent .1996;75:169-76.

12. Bishara SE ,Jakossen JR ,Abdulla EA, Garcia AF. Comparison of mesiodistal and buccolingual crown dimension of permanent teeth in 3 population from Egypt ,Mexico and United state. Am J orthod Dentofac orthop .1989;96:416-22.

13. Hashim HA, Murshid Z. Mesiodistal tooth width. comparison between male and female. part 1.Egyptian dent $J$.1993;39:34345.

14. Kellam GA. Tooth size and arch parameter, their relation to crowding of dention: com- 
parison between Navajo Indian and American Caucasian (MS thesis) .

15. Hashim HA ,Al-gamidi S. Tooth width and arch width in normal and malocclusion sample.J Con Temp Dent Practis .2005; 6(2):036-051.

16. Hatim NA. Estimation of the width of artificial anterior teeth in relation to the width of opposing teeth(a comparative study).Al-Rafidian Dent J 2002;1:27-31.

17. KassabNH .The relation of maxillary anterior teeth to the facial measurement and face form. Al-Rafidian Dent J 2005;1:1523.

18. Hasan NH. Dental aesthetic and facial measurement. Al-Rafidian Dent J 2005;1:614.

19. Howell SW. Cranial variation in man .astudy by multivariatiate analysis of pattern of difference among recent human popula- tions .papers of Peabody museum of archoeology and ethnology .1973;67:1-259 .

20. Carter GA, Macnamara JA. Longitudinaldental arch change in adult .Am $j$ orthod dentofac orthop $1998 ; 114: 88-99$.

21. Hsu BS .The nature of arch width difference of the anterior openbite .Am j orthod dentofac orthop $1998 ; 113: 344-50$.

22. Ferrario FR ,Sfroza C,Colombo A,Dellavia C,Demaggoio FR. Three dimensional hard tissue palatal size and shape in human adolescent and adult .Clin orthod Rres .2001;4:141-7.

23. Salman KA. The relationship between the maxillary arch width ,depth and circumference .AlRafidian dent $j$.spss 2001:401-10.

24. Ferrario VF , Sfroza C, Colombo A, Dellavia C,Demaggoio FR. Three dimensional hard tissue palatal size and shape:A10 year longitudinal evaluation in healthy adult .Int $j$ adult orthod orthganth surg .2002;17:51-8. 